\title{
Actuator Fault Estimation Based on LPV Unknown Input Observer for Induction Machine
}

\author{
Ahmed AMRANE ${ }^{1 *}$, Abdelkader LARABI ${ }^{2}$, Abdel AITOUCHE ${ }^{3}$ \\ ${ }^{1}$ National School of Technology, Diplomatic city, Dergana, Bordj El Kiffan, 16121, Algiers, Algeria, and \\ CRIStAL UMR CNRS 9189, University of Lille 1, Lille, 59650, France. \\ Ahmed.Amrane@univ-lille1.fr, amraneahmed@gmail.com (*Corresponding author) \\ ${ }^{1,2}$ Laboratory of Systems Electric and Industrial (LSEI), University of Science and Technology (USTHB), BP \\ 32, EI Alia, Bab- Ezzouar 16111, Algiers, Algeria. \\ larabiabdelkader@yahoo.fr \\ ${ }^{3}$ CRIStAL UMR CNRS 9189, Hautes études d'ingénieur HEI-Lille, Lille, 59046, France, \\ abdel.aitouche@yncrea.fr

\begin{abstract}
This paper presents an unknown inputs observer (UIO) applied to an induction machine (IM) in order to estimate the actuator faults and the state variables. Knowing that the machine used is highly nonlinear, a model based on LPV (linear parameter varying) system of the machine is used. Indeed, using the induction machine based on LPV model, we develop the structure of an observer where the actuator faults are the unknown inputs. The conditions for convergence are based on the Lyapunov theory that will ensure the stability. Based on the LMI (Linear Matrix Inequalities), the gains of the UIO subject to actuator faults are confirmed and then ensure the efficiency of our approach. The obtained results through simulations demonstrate the effectiveness of the proposed approach.
\end{abstract}

Keywords: Induction motor, Unknown inputs observer, Linear parameter varying (LPV), Linear Matrix Inequalities (LMI), Actuator faults.

\section{Introduction}

Due to its simplicity, low manufacturing cost and low maintenance, the induction cage machine has earned its leading position in applications requiring very high dynamic and static performance [24]. On the other hand, this simplicity is accompanied by a low starting torque and at constant frequency, it transmits a constant speed. Power electronics has recently emerged as a complex and multidisciplinary technology which brought the variable frequency, and also flow control to provide high torque [26]. Thanks to this, the asynchronous motor was able to take a step ahead.

This machine is not easy to control due to the nonlinearity of its dynamic model. Moreover, its state variables are not all measurable and it is under the influence of parametric variations. Therefore, a good control is necessary to guaranty the stability of its proper functioning. In the literature, most of the works deal with steady state vector control [8-10, 21, 25], Direct Torque Control (DTC) [19], Sliding Mode Control (SMC) $[13,27]$. In addition, nonlinear control applied to induction machine has been developed such in [14].

The advantages of such approaches are their robustness to disturbances and their simplicity of implementation. The major disadvantage of the set-up is that all controls rely on the reliability of the measurement provided by sensors, which makes these commands vulnerable. The occurrence of an output sensor fault and especially the actuator faults which directly affect them, hence the development of fault-tolerant controls is needed $[6,11,15]$.

The first step of active fault tolerant control is the detection and the location of faults. Fault detection uses up to now signal processing techniques [17], for operating phases, which highlights and localizes a dysfunction. Moreover, most of the techniques are based on observers $[1,23]$ or analytical redundancy based on parity space [3].

The second step, is the estimation of faults. Several works have been presented such us $[2$, 20]. However, the nonlinearity of the IM model makes the estimator's synthesis difficult. This problem is solved by separating the system into two subsystems, performing a linearization to obtain a linear model whose parameters depend on the rotation speed. Then a Linear Parameter Varying model (LPV) has been obtained and developed in several works. Reference [21] is dedicate to design a nonlinear method for a fault diagnosis method based on a polytypic linear parameter varying (LPV) formulation, and [12] is interested in a stability analysis of the double feed induction machine using LPVs. On the other hand, in [4] a design of a linear parameter varying model is used to control the speed of the shaft angle of 
an induction motor. Moreover, to eliminate the nonlinear character of the model, the authors of [16] use a Takagi-Sugeno approach for the design of an unmeasurable premise variable nonlinear observer for determining parameters inside a PEM Fuel Cell which is able to estimate the pressures and mass flow rates.

There are several methods developed for fault diagnosis and estimation, and the large challenge resides in the pairing between the LPV model and the estimator. A solution has been made by using observers with unknown input, which is considered one of the most effective observers, and it is used in [7] for state estimation applied to particular class of uncertain systems. Reference [22] deals with a nonlinear unknown input observer based on linear matrix inequality approach and propose an observer for the fault actuator detection and isolation scheme. Reference [5] deals with a nonlinear observer to detect for actuator fault (Exhaust Gas Recirculation) of diesel engines.

In this context, the main objective of this study is to put in place an observer with unknown input based on an LPV model that can efficiently estimate the faults of the actuators and the output states of the IM. This problem will then be translated into a convex optimization problem that will be solved using the Linear Matrix Inequalities (LMI) tools.

Section 2 deals with the presentation of the dynamic model of IM. The model given in section 2 is transformed in LPV model where the rotation speed represents the nonlinearity of LPV model. The LPV model will be used, in Section 4, to perform the synthesis and design of an observer with unknown inputs (faults and disturbance). In Section 5, simulation results shown the performance and the effectiveness of the proposed approach. Finally, section 6 concludes this study and presents the future works.

\section{Dynamic model of induction machine}

The current modelling approach of the asynchronous machine is based on the transformation of the three-phase system into a bi-phase system equivalent to Park.

Thus, in order to study the diagnosis of the asynchronous machine, both the stator variables and the rotor variables of the Park transformations are generally applied under assumptions on the magnetic circuits. It can also be asserted that the model is nonlinear as shown by the following equations $[9,11,19,21,26]$ :

Here, the stator and rotor current components with Flux and speed velocity are considered as state variables, the stator voltage components are considered as inputs, stator current components and rotation speed are considered as outputs as given in Equations (1)-(7). Then the induction machine model written in state space form is:

$$
\left\{\begin{array}{l}
\dot{x}_{1}=-\gamma x_{2}+\omega_{s} x_{2}+\alpha k x_{3}+p k x_{4} \omega+u_{1} \\
\dot{x}_{2}=-\omega_{s} x_{1}-\gamma x_{2}+\alpha k x_{4}-p k x_{3} \omega+u_{2} \\
\dot{x}_{3}=-\alpha L_{m} x_{1}-\alpha x_{3}+\left(\omega_{s}-p \omega\right) x_{4} \\
\dot{x}_{4}=-\alpha L_{m} x_{2}-\alpha x_{4}-\left(\omega_{s}-p \omega\right) x_{3}
\end{array}\right.
$$

In addition, the equation of the rotor speed is the following:

$\dot{\omega}=-\frac{K_{f}}{J} \omega+\psi\left(x_{2} x_{3}-x_{1} x_{4}\right)+-\frac{1}{J} C_{r}$,

where the state vector $x$ is given by:

$x=\left[\begin{array}{llll}x_{1} & x_{2} & x_{3} & x_{4}\end{array}\right]^{T}$

and:

$x_{1}=i_{d s} ; x_{2}=i_{q s} ; x_{3}=\phi_{d r} ; x_{4}=\phi_{q r}$.

$\beta=\frac{1}{\sigma L_{s}}, \gamma=\beta R_{s m}, k=\frac{L_{m}}{\sigma L_{r} L_{s}}, \psi=\frac{p L_{m}}{J T_{r}}, \alpha=\frac{1}{T_{r}}$

$x \in \mathfrak{R}^{n}$ is the state vector, $u \in \mathfrak{R}^{p}$ is the vector of control inputs and $y \in \mathfrak{R}^{m}$ is the output vector and variables are functions of time t. All vectors are in appropriate dimensions. The output vector is:

$y=\left[\begin{array}{ll}y_{2} & y_{1}\end{array}\right]^{T}=\left[\begin{array}{ll}x_{2} & x_{1}\end{array}\right]^{T}$

The input vector is:

$u=\left[\begin{array}{ll}u_{1} & u_{2}\end{array}\right]^{T}=\left[\begin{array}{ll}V_{d s} & V_{q s}\end{array}\right]^{T}$ 
The input function matrix $B$ can be written as:

$B=\left[\begin{array}{l}B_{1} \\ B_{2}\end{array}\right]=\left[\begin{array}{cccc}\frac{1}{\sigma L_{s}} & 0 & 0 & 0 \\ 0 & \frac{1}{\sigma L_{s}} & 0 & 0\end{array}\right]^{T}$

All parameters with their values can be found in the Nomenclature Table.

\section{Linear Parameter Varying (LPV) model of induction machine}

Reference is made to the nonlinear state-space model of a squirrel-cage IM considered in equation $1[4,18]$. Under the assumption of linear magnetic circuits, the electrical and mechanical state equations turn out to be:

$\dot{x}=A(x) \cdot x(t)+B \cdot u(t)$

where:

$A(x)=\left[\begin{array}{cccc}-\gamma & \omega_{s} & \alpha k & p k \omega \\ -\omega_{s} & -\gamma & -p k \omega & 0 \\ -\alpha L_{m} & 0 & -\alpha & \left(\omega_{s}-p \omega\right) \\ 0 & -\alpha L_{m} & -\left(\omega_{s}-p \omega\right) & -\alpha\end{array}\right]$

The rotor speed is considered as a varying parameter $\rho=\omega$, the equation (6) can be interpreted as the state equation of an LPV system with state $x=\left[\begin{array}{llll}x_{1} & x_{2} & x_{3} & x_{4}\end{array}\right]^{T}$. This state equation (6) can be rewritten in the usual form:

$$
\left\{\begin{array}{c}
\dot{x}(t)=A(\rho) \cdot x(t)+B \cdot u(t) \\
y=C \cdot x(t)
\end{array}\right.
$$

where:

$$
A(\rho)=A_{0}+\omega A_{i}
$$

with:

$$
A_{0}=\left[\begin{array}{cccc}
-\gamma & \omega_{s} & \alpha k & 0 \\
-\omega_{s} & -\gamma & 0 & \alpha k \\
-\alpha L_{m} & 0 & -\alpha & \omega_{s} \\
0 & -\alpha L_{m} & -\omega_{s} & -\alpha
\end{array}\right]
$$

and:

$$
\mathrm{C}=\left[\begin{array}{llll}
1 & 0 & 0 & 0 \\
0 & 1 & 0 & 0
\end{array}\right]
$$

Due to physical limits, we assume that all the components of the state are measurable or estimable and this allowed us to say that:

$$
\rho_{\text {min }} \leq \rho \leq \rho_{\text {max }}
$$

For this purpose the following variation interval is obtained:

$$
\left\{\begin{array}{l}
A_{1}=A_{\omega \min } \Rightarrow \rho_{1}=\frac{\omega-\omega_{\min }}{\omega_{\text {max }}-\omega_{\min }} \\
A_{2}=A_{\omega \max } \Rightarrow \rho_{2}=\frac{\omega_{\text {max }}-\omega}{\omega_{\max }-\omega_{\min }}
\end{array}\right.
$$

with:

$$
A_{\omega \min }=\left[\begin{array}{cccc}
0 & 0 & 0 & p k \omega_{\min } \\
0 & 0 & -p k \omega_{\min } & 0 \\
0 & 0 & 0 & -p \omega_{\min } \\
0 & 0 & p \omega_{\min } & 0
\end{array}\right]
$$

and:

$$
A_{\omega \max }=\left[\begin{array}{cccc}
0 & 0 & 0 & p k \omega_{\max } \\
0 & 0 & -p k \omega_{\max } & 0 \\
0 & 0 & 0 & -p \omega_{\max } \\
0 & 0 & p \omega_{\max } & 0
\end{array}\right]
$$

So we will have:

$$
A(\rho)=\sum_{i=1}^{r} \rho_{i} A_{i}=\rho_{1} A_{\omega \min }+\rho_{2} A_{\omega \max }
$$

and $\sum_{i=1}^{r} \rho_{i}=1$ is verified.

\section{UIO design for a nonlinear system (LPV model)}

\subsection{Description and preliminaries on the observer}

The method adopted in this article is related to the work carried out in [3] which deals with the design of fault detection and isolation (FDI) for an induction machine using Non-Linear Analytical Redundancy (NLAR) and the residuals are generated by analytical redundancy relation. As mentioned in the introduction, this work will focus on the estimation of the actuator fault and 
the state estimation. The scheme presented in Figure 1, gives a presentation of the principle of the observer with unknown input, where $U$ represents the command, $f$ the actuator fault, $d$ the perturbation, $\hat{f}$ and $\hat{x}$ are respectively the estimate of the fault and the estimate of the state, and $y$ the output of the machine $[5,7,22]$.

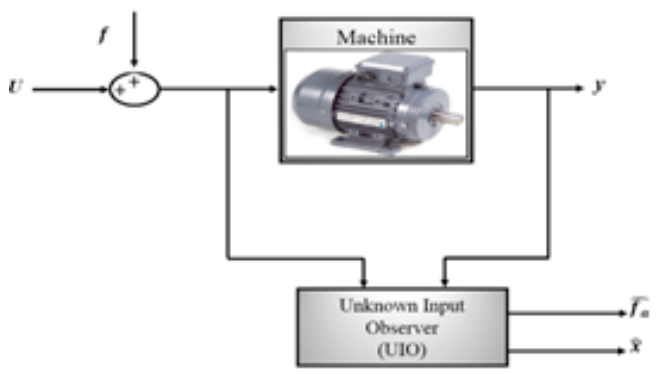

Figure 1. Schematic block of the approach

\subsection{Design of unknown input observer for IM}

Consider the variable system of linear parameters, described by an LPV model affected by actuator faults as follows:

$\left\{\begin{array}{l}\dot{x}(t)=A(\omega(t)) \cdot x(t)+B \cdot u(t)+F f(t) \\ y=C \cdot x(t)\end{array}\right.$

Where $f$ represents the faults vector. $A(\omega), B$ and $F$ are matrix of appropriate dimension.

The equation (14) can be rewritten as follows:

$\left\{\begin{array}{l}\dot{x}(t)=\sum_{i=1}^{r} \rho_{i} A_{i} \cdot x(t)+B . u(t)+F f(t) \\ y=C . x(t)\end{array}\right.$

Where $r$ is the number of varying parameter.

In this paper, in order to compute the unknown observer the following assumptions should be checked:

$>A_{i}$ is an invariant matrix over time.

$>\left(A_{i}, C\right)$ is observable.

$>C$ is full row rank.

$>f(t)$ are derived and bounded functions.

Based on the system (15), the unknown observer can be written as follows:

$\left\{\begin{array}{l}\dot{\mathrm{z}}(t)=\sum_{i=1}^{r} \rho_{i} N_{i} \cdot \mathrm{z}(t)+G \cdot u(t)+L y(t)+\hat{T f}(t) \\ \dot{x}(t)=z(t)-E x(t)\end{array}\right.$ where:

- $\quad z(t)$ is the state vector related to $x(t)$.

- $\quad N_{i}, G, L, T$ and $E$ are matrices of appropriate dimension and they satisfy the following conditions:

Knowing that the error $e(t)$ is defined such that:

$e(t)=x(t)-\hat{x}(t)$

then:

$e(t)=\left(\mathrm{I}_{2}+E C\right) x(t)-\mathrm{z}(\mathrm{t})$

Where $I_{2}$ is an identity matrix with appropriate dimensions.

Let $M=I_{2}+E C$ Then the expression of the error becomes:

$e(t)=M \cdot x(t)-\mathrm{z}(\mathrm{t})$

Therefore the dynamic error can be determined such that:

$\dot{e}(t)=M \dot{x}(t)-\dot{z}(\mathrm{t})$

$\dot{\mathrm{e}}(t)=\left[M \sum_{i=1}^{r} \rho_{i} A_{i}-\sum_{i=1}^{r} \rho_{i} N_{i} \mathrm{M}-L C\right] x(t)+\operatorname{MFf}(t)$

$+[M B-G] u(t)-T \hat{f}(t)+\sum_{i=1}^{r} \rho_{i} N_{i} \mathrm{e}(\mathrm{t})$

In order to compute the observer, he following conditions which guarantee the convergence of the estimation error should be hold:

$\left\{\begin{array}{l}M \sum_{i=1}^{r} \rho_{i} A_{i}-\sum_{i=1}^{r} \rho_{i} N_{i} \mathrm{M}-L C=0 \\ M B-G=0 \\ M F=T\end{array}\right.$

On the basis of the convergence conditions equation (19) one obtains:

$\dot{\mathrm{e}}(t)=\sum_{i=1}^{r} \rho_{i} N_{i} \mathrm{e}(\mathrm{t})+M F f(t)-T \hat{f}(t)$

The following change of variable is made:

$\tilde{f}(t)=f(t)-\hat{f}(t) \Rightarrow f(t)=\hat{f}(t)+\tilde{f}(t)$

Knowing that $M . F=T$ and based on equation (21), the dynamic error given in (20) becomes:

$\dot{\mathrm{e}}(t)=\sum_{i=1}^{r} \rho_{i} N_{i} \mathrm{e}(\mathrm{t})+T \tilde{f}(t)$ 
Based on the residual calculations, the following relationship is obtained:

$$
r_{e}=y(t)-\hat{y}(t)=C x(t)-C \hat{x}(t)=C e(t)
$$

The equation (24) gives the fault estimation:

$\hat{f}(t)=Q S\left[r_{e}-\int \delta r_{e} d t\right]$

where $Q$ is defined positive with appropriate dimension.

In order to make the LMIs feasible, the stability is checked by the following Lyapunov function which depends on the error of fault estimation:

$$
V=e^{T} P e+\frac{1}{\delta} \tilde{f}^{T} \mathrm{Q}^{-1} \tilde{f}
$$

By deriving the equation (25), the equation (26) is obtained:

$$
\dot{V}=\dot{e}^{T} P e+e^{T} P \dot{e}+\frac{1}{\delta} \dot{\tilde{f}}^{T} \mathrm{Q}^{-1} \tilde{f}+\frac{1}{\delta} \tilde{f}^{T} \mathrm{Q}^{-1} \dot{\tilde{f}}
$$

Knowing that $\dot{\tilde{f}}^{T} \tilde{f}=\tilde{f}^{T} \dot{\tilde{f}}$, we can obtain the following derivative Lyapunov function:

$$
\dot{V}=\dot{e}^{T} P e+e^{T} P \dot{e}+\frac{2}{\delta} \tilde{f}^{T} \mathrm{Q}^{-1} \dot{\tilde{f}}
$$

Replacing the value of the derivative of the error in equation (27):

$$
\begin{aligned}
& \dot{V}=\sum_{i=1}^{r} \rho_{i} \mathrm{e}^{T}\left[N_{i}^{T} P+P N_{i}\right] e+2 \tilde{f}^{T} T^{T} P e \\
& +\frac{2}{\delta} \tilde{f}^{T} \mathrm{Q}^{-1} \dot{\tilde{f}}
\end{aligned}
$$

By replacing equation (25) in the last equation, the expression of the derivative of the stability equation is obtained us follow:

$$
\begin{aligned}
& \dot{V}=\sum_{i=1}^{r} \rho_{i} \mathrm{e}^{T}\left[N_{i}^{T} P+P N_{i}\right] e+\frac{2}{\delta} \tilde{f}^{T} \mathrm{Q}^{-1} \dot{f} \\
& -\frac{2}{\delta} \tilde{f}^{T} S C \dot{e}
\end{aligned}
$$

Replacing equations (22) in the last equation:

$$
\begin{aligned}
& \dot{V}=\sum_{i=1}^{r} \rho_{i} \mathrm{e}^{T}\left[N_{i}^{T} P+P N_{i}\right] e+\frac{2}{\delta} \tilde{f}^{T} \mathrm{Q}^{-1} \dot{f} \\
& -\frac{2}{\delta} \tilde{f}^{T} S C \sum_{i=1}^{r} \rho_{i} N_{i} \mathrm{e}-\frac{2}{\delta} \tilde{f}^{T} S C T \tilde{f}
\end{aligned}
$$

\section{Lemma 01:}

Given a scalar $\mu$ and a positive symmetric matrix $\mathrm{P} 1$, the following equality is true [28]:

$$
2 x^{T} y \leq \frac{1}{\mu} x^{T} P_{1} x+\mu y^{T} P^{-1} y
$$

On the basis of lemma 01 , we can deduce the following inequality:

$\frac{2}{\delta} \tilde{f}^{T} Q^{-1} \dot{f} \leq \frac{1}{\delta}\left[\frac{1}{\mu} \tilde{f}^{T} P_{1} \tilde{f}+\mu Q^{-1 T} \dot{f}^{T} P^{-1} Q^{-1} \dot{f}\right]$

According to the proposed hypothesis, the derivative of the fault $f$ is bounded then: $\|\dot{f}\| \leq \alpha_{1}$ Such as $0 \prec \alpha_{1} \prec \infty$ and $\|\dot{f}\| \leq\left\|\dot{f}^{T}\right\|$

Then we have the following inequality

$\frac{2}{\delta} \tilde{f}^{T} Q^{-1} \dot{f} \leq \frac{1}{\delta} \frac{1}{\mu} \tilde{f}_{a}^{T} P_{1} \tilde{f}+\frac{\mu}{\delta} \alpha_{1}^{2} \lambda_{\max } Q^{-1 T} P^{-1} Q^{-1}$

In order to not charge the notation, we put:

$B=\frac{\mu}{\delta} \alpha_{1}^{2} \lambda_{\max } Q^{-1 T} P^{-1} Q^{-1}$

Replacing the latest results in equation (30):

$$
\begin{aligned}
& \dot{V} \leq \sum_{i=1}^{r} \rho_{i} \mathrm{e}^{T}\left[N_{i}^{T} P+P N_{i}\right] e+\frac{1}{\delta} \frac{1}{\mu} \tilde{f}^{T} P_{1} \tilde{f}+B \\
& -\frac{2}{\delta} \tilde{f}^{T} T^{T} \sum_{i=1}^{r} \rho_{i} N_{i} \mathrm{e}-\frac{2}{\delta} \tilde{f}^{T} S C T \tilde{f}
\end{aligned}
$$

We define the vector:

$\varepsilon=\left[\begin{array}{c}e \\ \tilde{f}\end{array}\right]^{T}$

We can write now the vector $\dot{V}$ as follows:

$\dot{V} \leq \sum_{i=1}^{r} \rho_{i} \varepsilon^{T} \psi_{i} \varepsilon+B$

Such as:

$\varepsilon=\left[\begin{array}{cc}N_{i}^{T} P+P N_{i} & * \\ \frac{1}{\delta} T^{T} P N_{i} & \frac{1}{\delta} \frac{1}{\mu} P_{1}-\frac{2}{\delta} P^{T} T^{T}\end{array}\right]$ 
Algorithm of Unknown Input Observer design

Step 1: Compute $H$ from the next equation then obtain the matrices $M, E$ :

$$
H=\left[\begin{array}{ll}
M & E
\end{array}\right]=\left[\begin{array}{ll}
I & 0
\end{array}\right]\left[\begin{array}{c}
I \\
-C
\end{array}\right]^{+}
$$

Step 2: Compute $G$ and $T$ from the next equations:

$$
G=M B ; T=M F
$$

Step 3: Solve the next $\boldsymbol{L M I}$ form equalities, obtain the matrices $P$, and calculate $K_{i}$ :

$$
\left[\begin{array}{cc}
N_{i}^{T} P+P N_{i} & * \\
-\frac{1}{\delta} T^{T} P N_{i} & \frac{1}{\delta} \frac{1}{\mu} P_{1}-\frac{2}{\delta} P^{T} T^{T}
\end{array}\right] \leq 0
$$

In order to resolve the $\boldsymbol{L M I}$ let: $\bar{K}_{i}=P \cdot K_{i}$ and we replace $N_{i}$ by the following equation:

$N_{i}=M \cdot A_{i}-K_{i} \cdot C$

Then we have the following inequality to solve:

$$
\left[\begin{array}{cc}
A_{i}^{T} M^{T} P+P A_{i} M-\bar{K}_{i} \cdot C-\bar{K}_{i}^{T} \cdot C^{T} & * \\
-\frac{1}{\delta} T^{T} P A_{i} M+\frac{1}{\delta} T^{T} P \bar{K}_{i} C & \frac{1}{\delta} \frac{1}{\mu} P_{1}-\frac{2}{\delta} P^{T} T^{T}
\end{array}\right] \leq 0
$$

Step 4: Compute matrices $N_{i,} L_{i}$ and $S_{i}$ from the equations (40), (41) and (42) respectively:

$L_{i}=K_{i}(I+C \cdot E)-M \cdot A_{i} \cdot E$

$S=T^{\prime} P C^{-1}$

\section{Application to Induction Machine}

We will simulate the model of the induction machine whose current outputs are considered as input variables for the observer has unusual inputs and the rotor speed is chosen as time variable as given in the section (3).

The computation will be done with the toolbox YALMIP, according to the equations of the observer and with the following conditions:
- The speed varies between a minimum value $\omega_{\min }=0$, and a maximum value $\omega_{\max }=157 \mathrm{rad} / \mathrm{s}$.

- We take $\mu=100$ and $\delta=10^{-4}$.

In order to demonstrate the effectiveness of the study method, a simulation of the dynamic behaviour of the machine are performed and the observer has been tested for two faults possible of the actuator, i.e. a fault in the stator direct voltage $\left(V_{d s}\right)$ and $\left(V_{q s}\right)$, the results are given in the figures (2-6) and this for the following conditions:

De will simulate only one actuator fault at a time, which will be around of $15 \%$ of the nominal values.

Test 1: state without fault.

Test 2: state with a quasi-square fault between $3 \mathrm{~s}$ and $7 \mathrm{~s}$.

Test 3: state with a variable fault between $0 \mathrm{~s}$ and $8 \mathrm{~s}$.

The matrix values obtained are as follows:

$A_{1}=\left[\begin{array}{cccc}-264.71 & 314.15 & 420.91 & 0 \\ -314.15 & -264.71 & 0 & 420.91 \\ 3.58 & 0 & -13.89 & 314.15 \\ 0 & 3.58 & 314.15 & -13.89\end{array}\right]$
$A_{2}=\left[\begin{array}{cccc}-264.71 & 314.15 & 420.91 & 9517.4 \\ -314.15 & -264.71 & 9517.4 & 420.91 \\ 3.58 & 0 & -13.89 & 0.2 \\ 0 & 3.58 & 0.2 & -13.89\end{array}\right]$

$B=\left[\begin{array}{cc}32.1898 & 0 \\ 0 & 32.1898 \\ 0 & 0 \\ 0 & 0\end{array}\right] ; C=\left[\begin{array}{cccc}1 & 0 & 0 & 0 \\ 0 & 1 & 0 & 0\end{array}\right] ; F=\left[\begin{array}{cc}1 & 0 \\ 0 & 1 \\ 0 & 0 \\ 0 & 0\end{array}\right]$

$M=\left[\begin{array}{cccc}0.25 & 0.25 & 0 & 0 \\ -0.25 & 0.25 & 0 & 0 \\ 0 & 0 & 1 & 0 \\ 0 & 0 & 0 & 1\end{array}\right] ; \mathrm{E}=\left[\begin{array}{rl}-0.75 & -0.25 \\ -0.25 & -0.75 \\ 0 & 0 \\ 0 & 0\end{array}\right]$

$N_{1}=\left[\begin{array}{cccc}-330.65 & 307.15 & 105.23 & -105.23 \\ 330.58 & -308.40 & -105.23 & 105.23 \\ 1.357 & -15.793 & -18.887 & 314.16 \\ -34.656 & 6.8448 & -314.16 & -13.887\end{array}\right]$ 
$N_{2}=\left[\begin{array}{cccc}-747.4 & 759.9 & 2484.6 & 2274.1 \\ 747.2 & -763 & -2484.6 & -2274.1 \\ 0.9 & -2.4 & -13.9 & 0.2 \\ 0.7 & 1.9 & 0.2 & -13.9\end{array}\right]$

$L_{1}=\left[\begin{array}{cc}171.81 & -14.73 \\ -172.11 & 15.03 \\ -0.705 & 4.288 \\ 10.375 & -6.79\end{array}\right] ; L_{2}=\left[\begin{array}{cc}389.19 & -232.11 \\ -389.91 & 232.84 \\ 2.756 & 0.826 \\ -0.644 & 4.226\end{array}\right]$

$G=\left[\begin{array}{cl}8.0475 & -8.0475 \\ -8.0475 & 8.0475 \\ 0 & 0 \\ 0 & 0\end{array}\right] ; T=\left[\begin{array}{cl}0.25 & -0.25 \\ -0.25 & 0.25 \\ 0 & 0 \\ 0 & 0\end{array}\right]$

$S=10^{3} \cdot\left[\begin{array}{cc}-2.0171 & -2.3658 \\ 2.0171 & 2.3658\end{array}\right] ; \mathrm{Q}=\left[\begin{array}{cc}0.33 & 0.06 \\ 0.06 & 0.33\end{array}\right]$

Figure 2 shows the evolution of currents $\left(I_{d s}\right.$ and $I_{q s}$ ) and the estimation of fault in the case of free system.
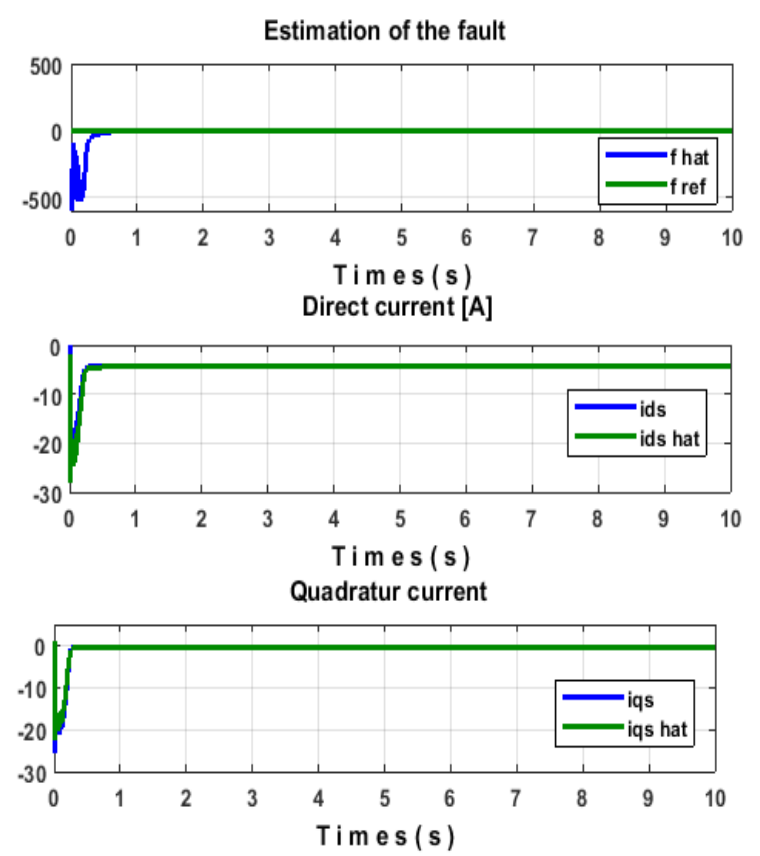

Figure 2. Free system: Estimation without actuator faults

Since the machine is initially stopped, a transient state less than half second is noted. Subsequently, a permanent regime is established and the observer follows the evolutions of the state variables and the fault.
Figure 3 shows the evolution of the reference and estimated fault, as well as the errors of estimating the states and the fault with actuator faults in $V_{d s}$. The results show that the errors converge to zero, which makes it possible to obtain good estimation results.

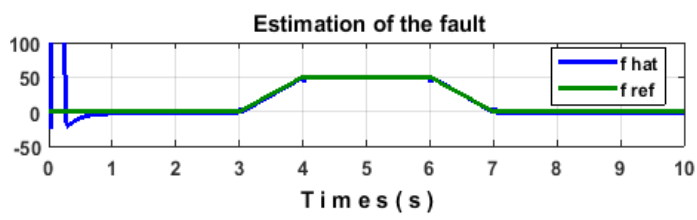

Estimation error of the fault

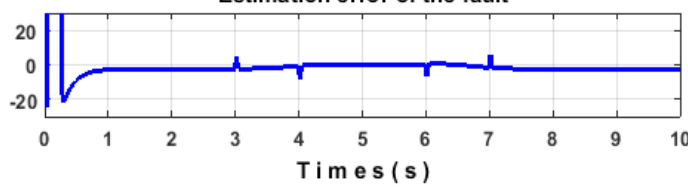

Estimation error of Direct current
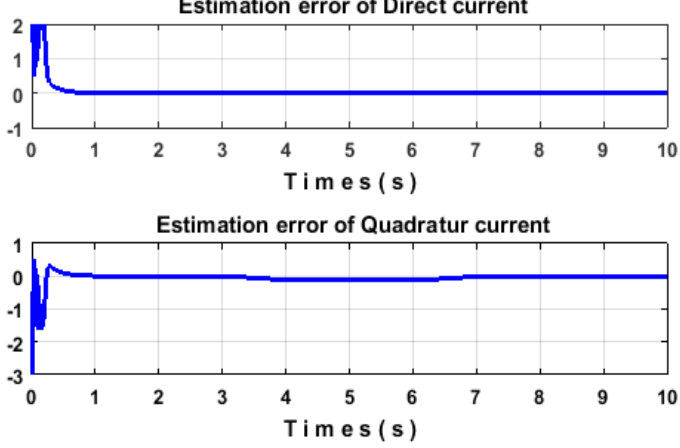

Figure 3. Estimation of square actuator faults in $V_{d s}$ The results obtained for a square error on $V_{q s}$ are presented in Figure.4, and it is noted that the error is well estimated and the estimation errors are around zero.
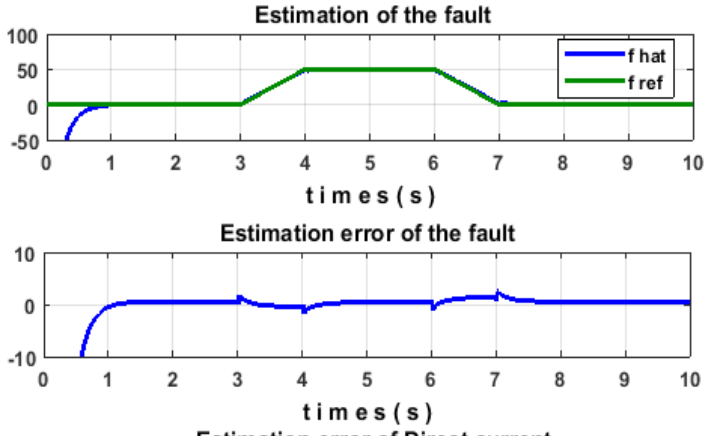

Estimation error of Direct current

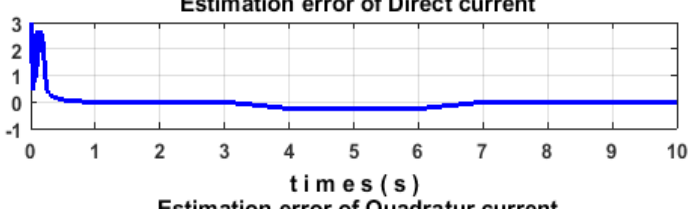

Estimation error of Quadratur current

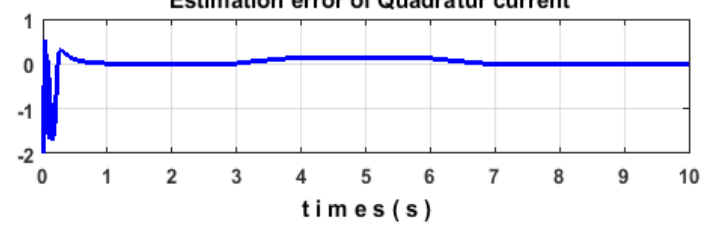

Figure 4. Estimation of square actuator faults in $V_{q s}$ 
From the results shown in Figures 5 and 6, it can be noted that for a variable fault on $V_{d s}$ and $V_{q s}$, we can say that there is no difference between the square fault and the variable fault. This confirms the results obtained in the preceding figures.
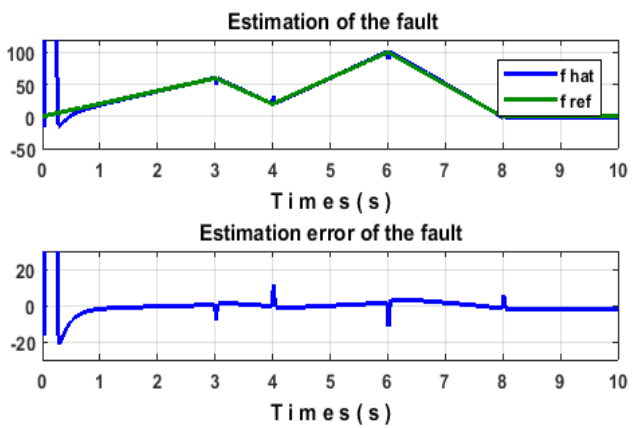

Estimation error of Direct current

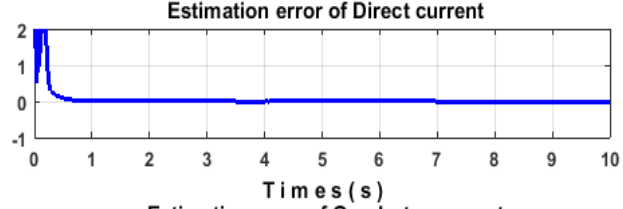

Estimation error of Quadratur current

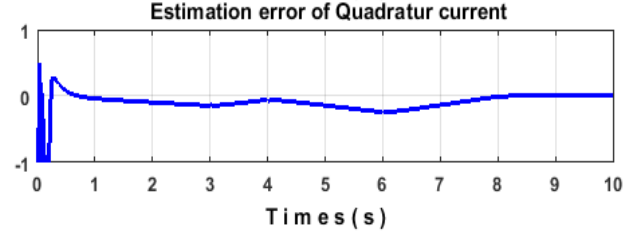

Figure 5. Estimation of variable actuator faults in $V_{d s}$

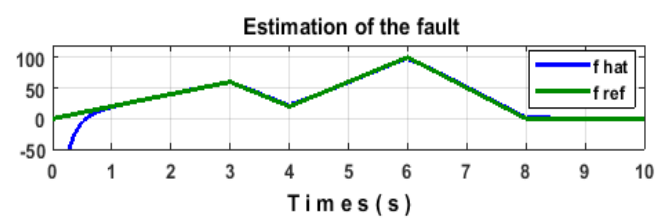

Estimation error of the fault

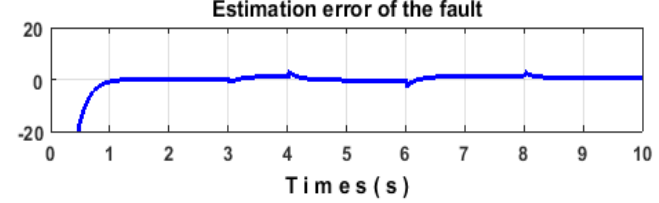

Estimation error of Direct current

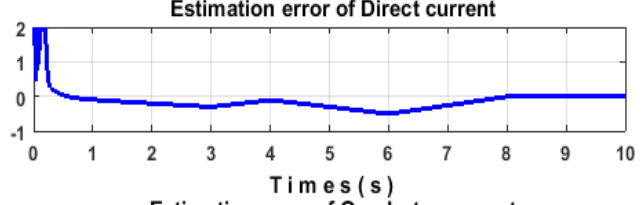

Estimation error of Quadratur current

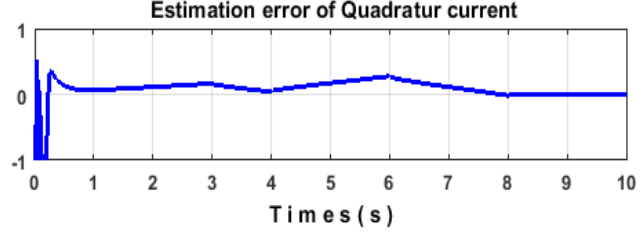

Figure 6. Estimation of variable actuator faults in $V_{q s}$

In the previous figures, we observe that the observer's estimated error values converge towards their steady-state references with short transitional regime and the estimation error tends to zero. We also notice that the output currents of the observer follow those of the machine and the error is almost zero. So the estimation by this method gives almost good results.

From these results, also we can say that the unknown inputs observer applied to an induction machine has very satisfactory performance.

\section{Conclusion}

The results obtained show our contribution to the problem of state and fault actuator of the induction machine modelled by a linear parameter varying model.

In order to obtain the observer gain by the resolution of LMIs, we choose a Lyapunov function which depends not only the error of estimation but also the error of fault estimation.

The estimation of faults and states has been reached despite the complexity of the system. On the basis of the results obtained, we can conclude that the approach also presents an interesting application in the field of fault-tolerant control design which will be the subject of future work in addition to the application of this study on a real machine.

\section{Acknowledgements}

This work was supported by the Ministry of Higher Education and Scientific Research of Algeria, the Research center in Computer Science, Signal and Automatic Control of Lille, France, (CRIStAL UMR CNRS 9189) and the graduate school of Engineering "Hautes etudes d'Ingenieur", Lille.

\section{Appendix A}

The parameters of the induction machine cage used are shown below:

\begin{tabular}{|l|l|l|}
\hline Rated power & $1.5 \mathrm{KW}$ & $P_{N}$ \\
\hline Nominal voltage & $220 / 380 \mathrm{~V}$. & $V_{N}$ \\
\hline Speed & $1420 \mathrm{rad} / \mathrm{min}$ & $\omega$ \\
\hline Nominal frequency & $50 \mathrm{~Hz}$ & $f$ \\
\hline Stator resistance & $4.85 \Omega$ & $R_{s}$ \\
\hline Rotor resistance & 3.805 & $R_{r}$ \\
\hline Stator cyclic inductance & $0.274 \mathrm{H}$. & $L_{s}$ \\
\hline Rotor Cyclic inductance & $0.274 \mathrm{H}$ & $L_{r}$ \\
\hline Cyclic mutual inductance & $0.258 \mathrm{H}$. & $L_{m}$ \\
\hline Number of pole pairs & 2 & $P$ \\
\hline Moment of Inertia & $0,031 \mathrm{Nms} / \mathrm{rad}$ & $J$ \\
\hline Friction & $0.008 \mathrm{Nm} \mathrm{s} / \mathrm{rad}$ & $K_{f}$ \\
\hline
\end{tabular}




\section{Appendix B}

\begin{tabular}{|l|l|}
\hline Synchronous Pulsation [rad/s]. & $\omega_{s}$ \\
\hline Electrical angular Pulsation [rad/s]. & $\omega_{r}$ \\
\hline Electromagnetic torque [N.m]. & $C_{e m}$ \\
\hline Resistive torque [N.m]. & $C_{r}$ \\
\hline Rotor time constant [s]. & $T_{r}$ \\
\hline The direct stator voltage & $V_{d s}$ \\
\hline The quadrature staor vltage & $V_{q s}$ \\
\hline The direct stator current & $i_{d s}$ \\
\hline The stator quadrature current & $i_{q s}$ \\
\hline The direct stator flow & $\Phi_{d r}$ \\
\hline The stator quadrature flow & $\Phi_{q r}$ \\
\hline
\end{tabular}

\section{REFERENCES}

1. A. Abbasi, J. Poshtan \& A. Moarefianpour. Decentralized Approach Based on Unknown Input Observers for Actuator Fault Detection and Isolation of a Class of Interconnected Nonlinear Systems. Studies in Informatics and Control, 25(4), 2016, pp. 453-460.

2. M Allouche, M Souissi, M Chaabane, D Mehdi \& F Tadeo. Takagi-Sugeno Fuzzy Observer Design for Induction Motors with Immeasurable Decision Variables: State Estimation and Sensor Fault Detection. International Journal of Computer Applications, 23(4), 2011, pp. 44-51.

3. A. Amrane, A. Larabi, \& A. Aitouche. Fault Detection and Isolation based on Nonlinear Analytical Redundancy applied to an Induction Machine. $6^{\text {th }}$ International Conference on Systems and Control, Batna, Algeria, 2017, pp. 293-298.

4. F. Blanchini, D. Casagrande, S. Miani \& U. Viaro. An LPV control scheme for induction motors, 51st IEEE Conference on Decision and Control (CDC), Maui, HI, 2012, pp. 7602-7607.

5. B. Boulkroune, A. Aitouche \& V. Cocquempot. Observer design for nonlinear parameter-varying systems: Application to diesel engines, International Journal of Adaptive Control and Signal Processing, 29(2), 2015, pp. 143-157.

6. O. Chenaru, D. Popescu \& D. Enache, L. Ichim. Fault-Tolerant Control System Implementation Based on Parameter Analysis. Studies in Informatics and Control, 25(2), 2016, pp. 227-236.
7. W. Jamel, A. Khedher, N. Bouguila, K. Benothman, State Estimation via Observers with Unknown Inputs: Application to a Particular Class of Uncertain Takagi-Sugeno Systems, Studies in Informatics and Control, 19(3), 2010, pp. 219-228.

8. J. Kan, K. Zhang \& Z. Wang. Indirect vector control with simplified rotor resistance adaptation for induction machines. IET Power Electronics, 8(7), 2015, pp. 1284-1294.

9. R. Kavitha, S. Nagaraju. Modeling and Implementation of fuzzy vector control for Induction motor Drive. International Journal of Engineering Research and General Science, 3(4), 2015, pp. 371-382.

10. N. Kobayashi, F. P. Wijaya, K. Kondo \& O. Yamazaki. Induction Motor Speed Sensorless Vector Control Using Mechanical Simulator and Disturbance Torque Compensation. IEEE Transactions on Industry Applications. 52(3), 2016, pp. 2323-2331.

11. H. Liu, Z. Mao, B. Jiang \& K. Zhang. Robust fault-tolerant control design for induction motor with faults and disturbances. $35^{\text {th }}$ Chinese Control Conference (CCC), Chengdu, 2016, pp. 6795-6800.

12. F.R. Lopez-Estrada, J.-C. Ponsart, D. Theilliol, Y. Zhang, \& C. Astorga-Zaragoza. LPV Model-Based Tracking Control and Robust Sensor Fault Diagnosis for a Quadrotor UAV. Journal of Intelligent \& Robotic Systems, 84, 2016, pp. 163-177.

13. M. Louri, A. Amrane \& L. Barazane. Comparison between the performances of variables structures control and the theory of synergetic on applied to the squirrel motor drives. 3rd International IEEE Conference on Systems and Control, Algiers, 2013, pp. 293-298.

14. M. Majdi Mansouri, N. Hazem Nounou \& N. Mohamed Nounou. Nonlinear control and estimation in induction machine using state estimation techniques. Systems Science \& Control Engineering. 2 (1), 2014, pp. 642-654.

15. H. Mekki, O. Benzineb, D. Boukhetala, M. Tadjine, \& M. Benbouzid. Sliding mode based fault detection, reconstruction and fault tolerant control scheme for motor systems. ISA Transactions, 57, 2015, pp. 340-351. 
16. S.C. Olteanu, A. Aitouche, L. Belkoura \& A. Jouni. Embedded P.E.M. Fuel Cell Stack Nonlinear Observer by means of a TakagiSugeno Approach. Studies in Informatics and Control. 24(1), 2015, pp. 61-70.

17. A. Ratni, C. Rahmoune \& D. Benazzouz. A new method to enhance of fault detection and diagnosis in gearbox systems. Journal of Vibroengineering. 19(1), 2017, pp. 176-188.

18. M. Rodrigues, M. Sahnoun, \& D. Theilliol, J-C. Ponsart. Sensor fault detection and isolation filter for polytopic LPV systems: A winding machine application. Journal of Process Control, 23(6, 2013, pp. 805-816.

19. J. Rodriguez, J. Pontt, C. Silva, S. Kouro \& H. Miranda. A novel direct torque control scheme for induction machines with space vector modulation. IEEE 35th Annual Power Electronics Specialists Conference, 2004, pp. 1392-1397.

20. T. Roubache, S. Chaouch \& M.S. Nait Said. Backstepping design for fault detection and FTC of an induction motor drives-based EVs. Automatica, 57(10), 2017, pp.736-748.

21. U. Saranya and S. Allirani. Model Reference Adaptive System based Speed Sensorless Control of Induction Motor using FuzzyPI Controller. International Journal of Computer Applications, 110(5), 2015, pp. 23-28.

22. M. Sharifuddin, C. Goutam \& B. Kingshook. Robust Unknown Input Observer for Nonlinear Systems and Its Application to Fault Detection and Isolation. Journal of Dynamic Systems Measurement and Control, 130 (4), 2008, pp. 1-5.
23. Q. Shen, B. Jiang and V. Cocquempot. Adaptive Fuzzy Observer-Based Active Fault-Tolerant Dynamic Surface Control for a Class of Nonlinear Systems with Actuator Faults. IEEE Transactions on Fuzzy Systems, 22 (2), 2014, pp. 338-349.

24. Y. Trachi, E. Elbouchikhi, V. Choqueuse \& M. E. H. Benbouzid. Induction Machines Fault Detection Based on Subspace Spectral Estimation. IEEE Transactions on Industrial Electronics, 63(9), 2016, pp.5641-5651.

25. V. Verma, C. Chakraborty, S. Maiti and Y. Hori. Speed Sensorless Vector Controlled Induction Motor Drive Using Single Current Sensor. IEEE Transactions on Energy Conversion, 28(4), 2013, pp. 938-950.

26. Y. Wang, T. Ito \& R. D. Lorenz. Loss Manipulation Capabilities of Deadbeat Direct Torque and Flux Control Induction Machine Drives. IEEE Transactions on Industry Applications, 51(6), 2015, pp. 45544566.

27. H. Xu, F. Zhao, W. Cong \& W. Peng. Study of a New Rotor Flux Estimator for Induction Machine Based on Sliding Mode Control. IEEE Vehicle Power and Propulsion Conference (VPPC), Hangzhou, 2016, pp.1-5.

28. Y. Zhang, J. Jiang, Bibliographical review on reconfigurable fault-tolerant control systems, Annual Reviews in Control, 32(2), 2008, pp. 229-252. 